\section{SMGrsup}

\section{SM Journal of Orthopedics}

\section{Article Information}

Received date: Sep 28, 2017

Accepted date: Oct 16, 2017

Published date: Oct 25, 2017

\section{*Corresponding author}

Badara Dembélé, Orthopedic and Trauma Unit, CHU Aristide Le Dantec, Dakar, Senegal, West Africa, BP: 3001 Dakar, Tel: 00 (221) 775418514; Fax: 00 (221) 338342107; E-mail: badaradembele81@gmail.com

\section{Distributed under Creative Commons} CC-BY 4.0

Keywords Pseudarthrosis; Delayed consolidation; Sacrum; Medico; Physical therapy

\title{
Pseudarthrosis or Delayed Consolidation of the Sacrum (Diagnostic and Therapeutic Difficulties) - About a Case and Review of the Literature
}

\author{
Dembélé B*, Diouf AB, Daffé M, Gueye AB, Sarr L, Nguessie I, Sané AD, Coulibaly \\ NF and Diémé CB \\ Department of Orthopedics Traumatology, CHU Aristide Le Dantec, Senegal
}

\section{Abstract}

Introduction: Isolated fractures of the sacrum are rare and in principle related to a direct posterior shock. The occurrence of a pseudarthrosis of a sacrum associated with neurological disorders is an exceptional eventuality.

Case: Female subject 38 , victim of a traffic accident occurring 3 months previously, consulted for a neglected trauma of the left hemi pelvis with relative functional impotence of the lower left limb. Clinical examination resulted in a painful lameness with a makeshift cane, pain in inguinal palpation and mobilization of the left hip. The gluteus medius muscle was rated at 0 . There were no sphincteric disorders.

Observations: The standard X-ray showed a fracture of the left ischiopubic branch. At CT, there was also a vertical fracture of the left hemi-sacrum passing through the sacral holes and a fracture of the anterior column of the homolateral acetabulum.

The electromyogram showed a left L5 and S1 radiculopathy, a truncular involvement of the SPI (myelinic type) and of the left SPE (axonal type).

At 6 months of follow-up and after a medico-physical treatment (analgesic of pallium-Il, vitamino-therapy $B$ and functional rehabilitation); the patient fully recovered with pain only squatting and a gluteus medius to 5 .

Results and Conclusion: In traumatology of the pelvis, the standard images are often ill-readable, hence the interest of CT. In the absence of displacement and / or root compression, functional treatment is mandatory. The occurrence of a pseudarthrosis associated or not with irreducibility or a persistence of the neurological syndrome indicates a surgical approach.

\section{Introduction}

It is usually secondary to non-surgical treatment of sacral fracture or inappropriate surgical treatment. Pseudarthrosis of the sacrum causes severe clinical signs such as severe chronic pain, discomfort at sitting and a significant restriction of activity level, neurological deficits of the sacral roots, static and dynamics of the pelvis especially in women. The treatment of this complication is essentially surgical, but this can be accompanied by vascular, nervous and infectious complications.

\section{Observation}

It is a 38-year-old woman with no previous pathological history who was received second hand at 3 months of a closed pelvic trauma following a road traffic accident that had occurred $504 \mathrm{~km}$ from the capital. She was a passenger of a public transport vehicle that reportedly struck a bridge and then flipped over. Neither notion of wearing seat belts in the car, nor triggering airbags to collusion.

She had a painful lameness requiring the help of a makeshift cane, a spontaneously painful left hip and mobilization as well as functional impotence of the lower left limb.

He had no functional sphincter involvement or sacral roots from S1 to S5.

The morphological assessment showed a fracture of the left ischiopubic branch with a suspected fracture of the left hemi-sacrum with a vertical line (Figure 1).

Computed Tomography (CT) was used to confirm this fracture of the left hemi-pelvis passing through the sacral holes of S1 and S2 (Figure 2) and fracture of the anterior column of the left acetabulum.

The electromyogram showed (Figure 3): left L5 and S1 radiculopathy with truncal involvement of SPI (myelin type) and left SPE (axonal type). 


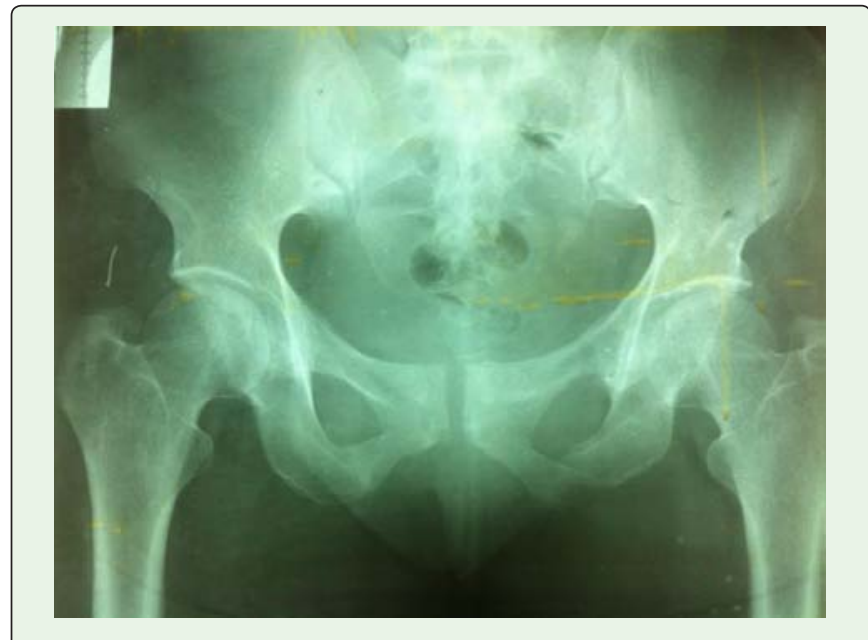

Figure 1: X-ray of the pelvis face 3 months.

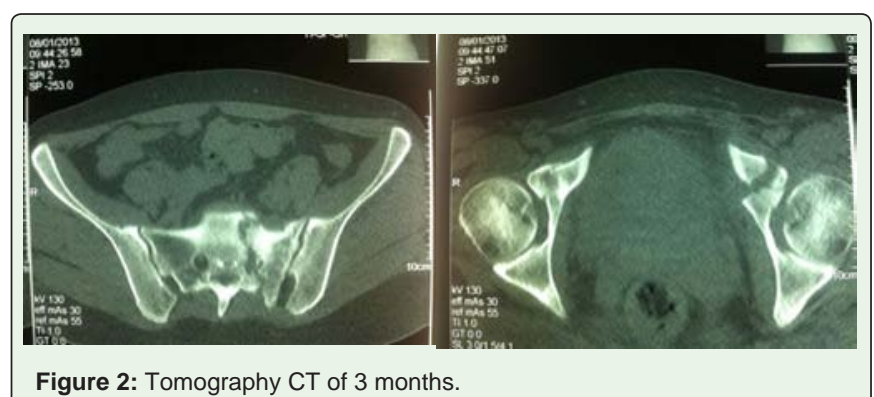

Figure 2: Tomography CT of 3 months.

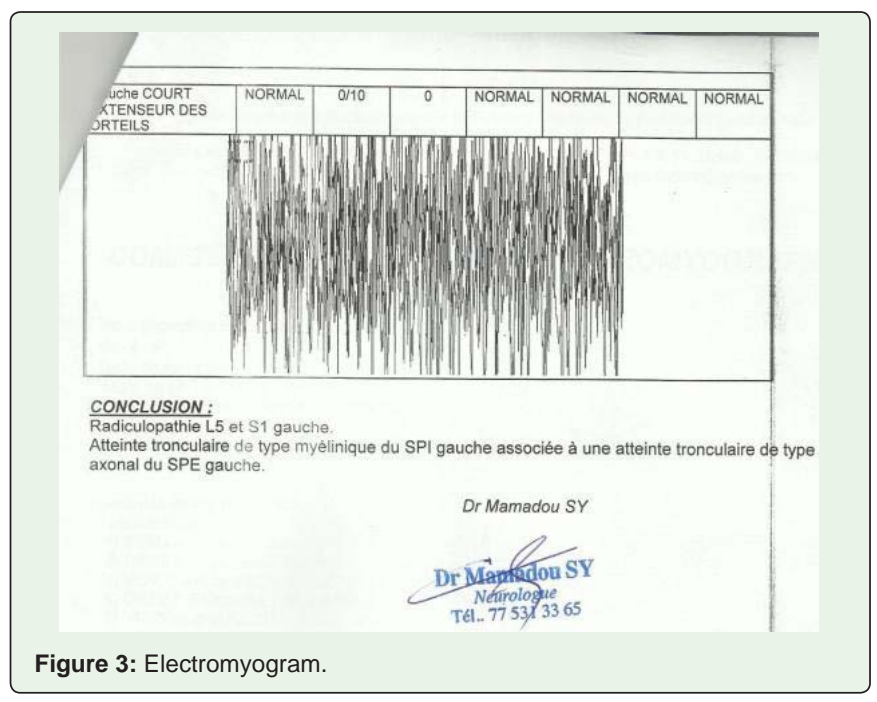

In view of the refusal of a proposed surgical treatment, a medicophysical treatment based on antalgic pallium-II, neuroregenerator and functional rehabilitation sessions (type of empowerment, muscle strengthening, proprioception and nociception).

Our patient was reviewed at 6 months, 12 months and 5 years post - therapy with a complete regression that was objectified in the clinic by: pain only squatting, autonomous walking without limping, buttocks rated at 5 .

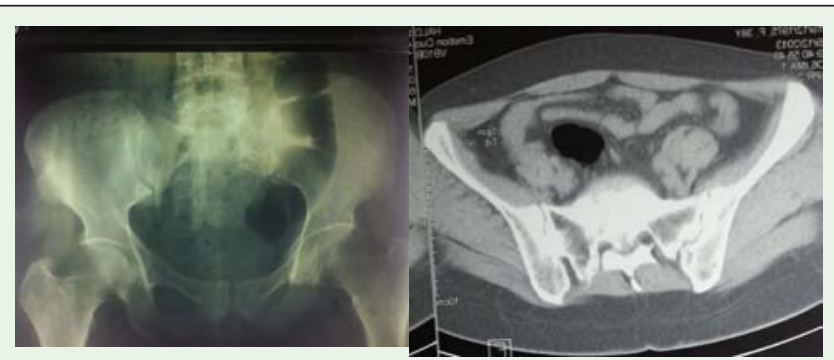

Figure 4: Control imaging after we take care of.

At the standard radiography of control confirmed by CT, we found: A consolidation of fractures of the anterior wall of the acetabulum and the ischiopubic branch as well as a fusion at the level of the left sacral line (Figure 4).

\section{Discussion}

The diagnostic errors of the pseudarthrosis of the sacrum and its management among, which the medico-physical treatment will be discussed:

Pseudarthrosis of the sacrum is a rare clinical condition. We discuss in this work, the diagnostic errors, and the treatment of this complication as well as the place of the medico-functional treatment in its management.

This clinical condition is often under diagnosed due to the difficulty of visualizing the fracture trait when interpreting standard radiography [1,2]. Although some clinical signs such as severe chronic pain, seated discomfort, walking abnormalities, and a significant restriction in the level of activity of patients with a history of pelvic trauma [3-6] should lead us to perform a CT scan of the pelvis that confirms the diagnosis.

As in all the literature, a fracture of the sacrum consolidates within a maximum of 2 months [7]. The appearance of a primary callus is done during the first month following the trauma. Our case confirms the conclusions of the literature both in the circumstances of discovery and in the diagnostic confirmation.

It is secondary in all series of the literature as well as in our case to a non-surgical treatment of a lateral fracture of a hemi sacrum except in the series of Westphal [2] where it is on the median line.

Several modalities of therapeutic management of this pseudarthrosis have been described in the literature $[1,3-5]$. The gold standard is represented by the open hearth using a direct posterior approach followed by an excision of the scar tissue, curettage of the banks, a bone graft then an auto or hetero-fixing either by a plate of reconstruction either by a simple compressive screwing $[4,6]$. Although bone autograft is the gold standard for this technique, few writings have been found describing this approach. Lee in his series uses a posterior approach for grafting [4]. This technique has led to a consolidation of pseudarthrosis in open-cure series $[4,8,9]$ with satisfactory results in more than $85 \%$ of cases [9] but with complications in approximately $20 \%$ of the cases of pseudarthrosis treated by this technique. On the other hand, it exposes to infectious risks, nervous lesions, blood loss at the time of admission and lengthening of the operative time $[4,5,9,10]$. 
The treatment of pseudarthrosis by closed-screw screwing constitutes an alternative which offers several advantages, namely reduction and stabilization without broad exposure of the focus [11]. Their consolidation rate is similar to that of open cure as reported in the literature $[5,11]$. However, this technique is more appropriate in cases of pseudarthrosis with little or no displacement and without neurological lesion as well as in patients with deformities of the posterior pelvis.

But whatever the surgical method used, it allows a clear improvement of the clinical symptoms which prompted the patient to consult. However, in some of the series most patients continued to suffer from pain during squatting $[1,2,4,5]$.

Our technique, namely the medical-physical treatment combining the prescription of analgesic pallium-II, neuroregenerator accompanied by functional rehabilitation to the type of physiotherapy plus balneotherapy can be a good alternative. Because it has allowed us to have a clear improvement in the symptomatology as well as a consolidation of this pseudarthrosis by micro-movements were generated at the level of the focus. On the other hand, we have not found in the literature this technique in case of management of a pseudarthrosis. This conservative treatment has the advantages of not opening the focus of the non-steroid, therefore not the risks of open cure (no blood loss, no risk of infection or risk of nerve damage and no surgical wound). It does not require any specific technical platform apart from a functional rehabilitation service and qualified trained rehabilitators. And our patient has only a small squatting gene, as described in all series [2-5].

\section{Conclusion}

Pseudarthrosis or delayed consolidation of a fracture of the sacrum constitutes a rare lesion that occurs on pathological bone or in osteopenia. But one should always think of it in case of severe chronic pain associated with a discomfort in sitting position and a significant restriction of the level of activity more anomaly of a patient's walk to a history of pelvic trauma as reported in the literature. It is often secondary to initial non-surgical treatment.

Therapeutic management is essentially surgical with both open and closed osteosynthesis, using screws or a reconstruction plate.
Treatment will most often be surgical; however, the functional (medico-physical) treatment traditionally described for the fracture of the sacrum involves, in the case of pseudarthrosis or delayed consolidation of the sacrum, a risk compared to bed rest which is dangerous thromboembolic and in a patient who may remain confined to the bed.

In our therapeutic approach, we excluded him, preferring, among other things, a setting-up and immediate empowerment.

\section{References}

1. Ebrahim NA, Ashok B, Frank W. Non-union of pelvic fracture. J Trauma 1998. 44: 202-204.

2. Westphal T, Piatek S, Winckler S. Pseudarthrosis of an occult fracture in zone-III of the sacrum. Unfallchirurg. 1999; 102: 493-496.

3. Huegli RW, Messmer P, Jacob AL, Regazzoni P, Styger S, Gross T. Delayed union of a sacral fracture: Percutaneous navigated autologous cancellous bone grafting and screw fixation. Cardivasc Intervent Radiol. 2003; 26: 502 505 .

4. Lee SY, Takahiro N, Yoshitada S, Masahiko M, Nishida K, Ryosuke K, et al. Sacral Fracture Nonunion Treated by Bone Grafting through a Posterior Approach. Casa Rep Orthop. 2013; 2013: 1-5.

5. Mears DC, John HV. In situ fixation of pelvic non-unions following pathologic and insufficiency fractures. J Bone Joint Surg Am. 2002; 84: 721-728.

6. Van Der Bosch EW, Van der Kleyn R, Marieke CMA Van Zwienen, Arie B Van Vugt. Nonunion of unstable fractures of the pelvis. Euro J Trauma. 2002; 28:100-103.

7. Bauer P. Guide pratique des urgences traumatologiques. Livre pour l'interne de garde aux urgencies. 2000: 92-93.

8. De Boeckh, Yde P, Opdecam P. Non-union of a sacral fracture treated by bone graft and internal fixation. Injury. 1995; 26: 65-66.

9. Matta JM, Dickson KF, Markovich GD. Surgical treatment of pelvic nonunions and malunions. Clin Orthop Relat Res. 1996; 329: 199-206.

10. Gautier E, Régent D, Paul JP, Pere P, Claudon M. Fracture par insuffisance osseuse du sacrum. J Radiol. 1987: 433-440.

11. Chip Routt ML, Peter S. Closed reduction and percutaneous skeletal fixation of sacral fractures Clin Orthop Relat Res. 1996; 329: 121-128. 